\title{
THE 24 MICRON SOURCE COUNTS IN DEEP SPITZER SPACE TELESCOPE SURVEYS ${ }^{1}$
}

\author{
C. Papovich, ${ }^{2}$ H. Dole ${ }^{3}$ E. Egami, ${ }^{2}$ E. Le Floc'h, ${ }^{2}$ P. G. Pérez-González, ${ }^{2}$ A. Alonso-Herrero, ${ }^{2,4}$ \\ L. Bai, ${ }^{2}$ C. A. Beichman, ${ }^{5}$ M. Blaylock, ${ }^{2}$ C. W. Engelbracht, ${ }^{2}$ K. D. Gordon, ${ }^{2}$ D. C. Hines, ${ }^{2,6}$ \\ K. A. Misselt, ${ }^{2}$ J. E. Morrison, ${ }^{2}$ J. Mould $^{7}$ J. Muzerolle, ${ }^{2}$ G. Neugebauer, ${ }^{2}$ P. L. Richards, ${ }^{8}$ \\ G. H. Rieke, ${ }^{2}$ M. J. Rieke, ${ }^{2}$ J. R. Rigby, ${ }^{2}$ K. Y. L. Su, ${ }^{2}$ and E. T. Young ${ }^{2}$ \\ Received 2004 March 25; accepted 2004 May 17
}

\begin{abstract}
Galaxy source counts in the infrared provide strong constraints on the evolution of the bolometric energy output from distant galaxy populations. We present the results from deep $24 \mu \mathrm{m}$ imaging from Spitzer surveys, which include $\approx 5 \times 10^{4}$ sources to an $80 \%$ completeness of $\simeq 60 \mu \mathrm{Jy}$. The $24 \mu \mathrm{m}$ counts rapidly rise at near-Euclidean rates down to $5 \mathrm{mJy}$, increase with a super-Euclidean rate between 0.4 and $4 \mathrm{mJy}$, and converge below $\sim 0.3 \mathrm{mJy}$. The $24 \mu \mathrm{m}$ counts exceed expectations from nonevolving models by a factor of $\gtrsim 10$ at $S_{\nu} \sim 0.1 \mathrm{mJy}$. The peak in the differential number counts corresponds to a population of faint sources that is not expected from predictions based on $15 \mu \mathrm{m}$ counts from the Infrared Space Observatory. We argue that this implies the existence of a previously undetected population of infrared-luminous galaxies at $z \sim 1-3$. Integrating the counts to $60 \mu \mathrm{Jy}$, we derive a lower limit on the $24 \mu \mathrm{m}$ background intensity of $1.9 \pm 0.6 \mathrm{nW} \mathrm{m}^{-2} \mathrm{sr}^{-1}$ of which the majority $(\sim 60 \%)$ stems from sources fainter than $0.4 \mathrm{mJy}$. Extrapolating to fainter flux densities, sources below $60 \mu \mathrm{Jy}$ contribute $0.8_{-0.4}^{+0.9} \mathrm{nW} \mathrm{m}^{-2}$ $\mathrm{sr}^{-1}$ to the background, which provides an estimate of the total $24 \mu \mathrm{m}$ background of $2.7_{-0.7}^{+1.1} \mathrm{nW} \mathrm{m}^{-2} \mathrm{sr}^{-1}$.
\end{abstract}

Subject headings: cosmology: observations - galaxies: evolution — galaxies: high-redshift galaxies: photometry — infrared: galaxies

Online material: machine-readable table

\section{INTRODUCTION}

From the first detections of infrared (IR) luminous galaxies, it was clear that they represent phenomena not prominent in optically selected galaxy surveys (e.g., Rieke \& Low 1972; Soifer et al. 1987). Locally, galaxies radiate most of their emission at UV and optical wavelengths, and only about onethird at IR wavelengths $(5-1000 \mu \mathrm{m}$; Soifer \& Neugebauer 1991). IR number counts from the Infrared Space Observatory (ISO) indicate that the IR-luminous sources have evolved rapidly, significantly faster than has been deduced from optical surveys, which implies that IR-luminous galaxies make a substantial contribution to the cosmic star formation rate density (e.g., Elbaz et al. 1999; Franceschini et al. 2001).

The detection of the cosmic background by the Cosmic Background Explorer ( $C O B E$ ) at IR wavelengths shows that the total far-IR emission of galaxies in the early universe is greater than that at optical and UV wavelengths (Fixsen et al. 1998; Hauser et al. 1998; Madau \& Pozzetti 2000; Franceschini et al. 2001), which suggests that a large fraction of stars have

\footnotetext{
${ }^{1}$ This work is based on observations made with the Spitzer Space Telescope, which is operated by the Jet Propulsion Laboratory, California Institute of Technology, under NASA contract 1407.

2 Steward Observatory, University of Arizona, 933 North Cherry Avenue, Tucson, AZ 85721; papovich@as.arizona.edu.

${ }^{3}$ Institut d'Astrophysique Spatiale, bat 121, Université Paris Sud, F-91405 Orsay Cedex, France.

4 Departamento de Astrofísica Molecular e Infrarroja, IEM, CSIC, Serrano 113b, 28006 Madrid, Spain.

5 Michelson Science Center, California Institute of Technology, Pasadena, CA 91109.

${ }^{6}$ Space Science Institute, 4750 Walnut, Suite 205, Boulder, CO 80301.

7 National Optical Astronomy Observatory, 950 North Cherry Avenue, Tucson, AZ 85719.

${ }^{8}$ Department of Physics, University of California, Berkeley, CA 94720.
}

formed in IR-luminous phases of galaxy activity (Elbaz et al. 2002). Studies from $I S O$ at 15 and $170 \mu \mathrm{m}$ have inferred that the bulk of this background originates in discrete sources with $z \lesssim 1.2$ (Dole et al. 2001; Elbaz et al. 2002). However, the peak in the cosmic IR background extends to $\sim 200 \mu \mathrm{m}$ (Fixsen et al. 1998; Hauser \& Dwek 2001). The spectral energy distributions (SEDs) of IR-luminous galaxies $\left(L_{\mathrm{IR}} \sim 10^{11}-10^{12} L_{\odot}\right)$ peak between 50 and $80 \mu \mathrm{m}$ (Dale et al. 2001). If these objects constitute a major component of the cosmic IR background, then it follows there is a significant population of IR-luminous galaxies at $z \sim 1.5-3$, distances largely unexplored by ISO.

The mid-IR $24 \mu \mathrm{m}$ band on the Multiband Imaging Photometer for Spitzer (MIPS; Rieke et al. 2004) is particularly well suited to studying distant IR-luminous galaxies. Locally, the mid-IR emission from galaxies relates almost linearly with the total IR luminosity over a range of galaxy types (e.g., Spinoglio et al. 1995; Chary \& Elbaz 2001; Roussel et al. 2001; Papovich \& Bell 2002), and there are indications this holds at higher redshifts (e.g., Elbaz et al. 2002). Because the angular resolution of Spitzer is significantly higher for the $24 \mu \mathrm{m}$ band relative to 70 and $160 \mu \mathrm{m}$, the $24 \mu \mathrm{m}$ confusion limit lies at fainter flux densities. This allows us to probe the IR emission from many more sources and at higher redshifts than with the MIPS longer wavelength bands (e.g., Papovich $\&$ Bell 2002; Dole et al. 2003). Here we present the number counts of $\approx 5 \times 10^{4}$ sources detected at $24 \mu \mathrm{m}$ in deep Spitzer surveys, and we suggest that the faint Spitzer detections probe a previously undetected population of very luminous galaxies at high redshifts. Where applicable, we assume $\Omega_{m}=0.3$, $\Omega_{\Lambda}=0.7$, and $H_{0}=70 \mathrm{~km} \mathrm{~s}^{-1} \mathrm{Mpc}^{-1}$.

\section{THE DATA AND SOURCE SAMPLES}

The data used in this work stem from early Spitzer characterization observations and from time allocated to the MIPS 
TABLE 1

Properties of DeEp Spitzer Fields

\begin{tabular}{|c|c|c|c|c|c|c|c|c|}
\hline $\begin{array}{l}\text { Field } \\
\text { (1) }\end{array}$ & $\begin{array}{c}\text { R.A. } \\
\text { (J2000.0) } \\
(2)\end{array}$ & $\begin{array}{c}\text { Decl. } \\
(\mathrm{J} 2000.0) \\
(3)\end{array}$ & $\begin{array}{c}\left\langle I_{\nu}\right\rangle \\
\left(\mathrm{MJy} \mathrm{sr}^{-1}\right) \\
(4)\end{array}$ & $\begin{array}{c}\text { Area } \\
\left(\operatorname{arcmin}^{2}\right) \\
(5)\end{array}$ & $\begin{array}{c}\left\langle t_{\exp }\right\rangle \\
\text { (s) } \\
(6)\end{array}$ & $\begin{array}{c}C_{80 \%} \\
(\mu \mathrm{Jy}) \\
(7)\end{array}$ & $\begin{array}{c}N\left(>C_{80 \%}\right) \\
\left(\operatorname{arcmin}^{-2}\right) \\
(8)\end{array}$ & $\begin{array}{c}N(>300 \mu \mathrm{Jy}) \\
\left(\operatorname{arcmin}^{-2}\right) \\
(9)\end{array}$ \\
\hline Marano ............ & $03 \quad 1352$ & -551523 & 19.7 & 1296 & 236 & 170 & 2.0 & 0.9 \\
\hline CDF-S ............ & 033228 & -274830 & 22.5 & 2092 & 1378 & 83 & 4.5 & 0.7 \\
\hline EGS & 141600 & +524850 & 19.5 & 1466 & 450 & 110 & 3.4 & 0.7 \\
\hline Boötes ........... & 143206 & +341648 & 22.7 & 32457 & 87 & 270 & 1.0 & 0.8 \\
\hline ELAIS ............ & 160952 & +545500 & 18.2 & 130 & 3232 & 61 & 5.7 & 0.6 \\
\hline
\end{tabular}

Notes.-Col. (1): Field name. Col. (2): Right ascension, in units of hours, minutes, and seconds. Col. (3): Declination, in units of degrees, arcminutes, and arcseconds. Col. (4): Mean $24 \mu \mathrm{m}$ background. Col. (5): Areal coverage. Col. (6): Mean exposure time. Col. (7): $80 \%$ completeness limit. Col. (8): Source density with $S_{\nu}>C_{80} \%$. Col. (9): Source density with $S_{\nu}>300 \mu \mathrm{Jy}$.

Guaranteed Time Observers (GTOs). The images were obtained in five individual fields with large angular separation on the sky in order to minimize cosmic-variance biases from large-scale structure, and with high-quality ancillary data at other wavelengths. The area and depth of each field vary substantially to adequately sample source densities at all flux levels with high statistical significance. Table 1 lists their properties and $24 \mu \mathrm{m}$ source densities. The MIPS fields used here subtend the largest areas $\left(\simeq 10.5 \mathrm{deg}^{2}\right)$ and widest range in flux density (50-0.06 mJy) available to date from the Spitzer mission and therefore are the premier data set for studying the mid-IR source counts.

The MIPS $24 \mu \mathrm{m}$ images were processed with a custom data analysis tool, developed by the MIPS GTOs (Gordon et al. 2004). The measured count rates are corrected for dark current, cosmic rays, and flux nonlinearities, and then divided by flat fields for each unique MIPS scan-mirror position. Images are then corrected for geometric distortion, co-added, and mosaicked. The final mosaics have a pixel scale of $\simeq 1$ 1"25 pixel $^{-1}$, with a point-spread function (PSF) FWHM of $\simeq 6^{\prime \prime}$.

We performed source detection and photometry using a set of tools and simulations. Briefly, we first subtract the image background using a median filter roughly 4 times the size of source apertures (see below) and use a version of the DAOPHOT software (Stetson 1987) to detect point sources. We filter each image with a Gaussian with an FWHM that is equal to that of the MIPS $24 \mu \mathrm{m}$ PSF and identify positive features in $10^{\prime \prime}$ diameter apertures above some noise threshold. We then construct empirical PSFs using 20-30 bright sources in each image, and we optimally measure photometry by simultaneously fitting the empirical PSFs to all sources within $\simeq 20^{\prime \prime}$ of nearby object centroids. The source photometry corresponds to the flux of these PSF apertures within a diameter of 37.4 , and we apply a multiplicative correction of 1.14 to account for light lost outside these apertures.

To estimate completeness and photometric reliability in the $24 \mu \mathrm{m}$ source catalogs, we repeatedly inserted artificial sources into each image with a flux distribution approximately matching the measured number counts. We then repeated the source detection and photometry process and compared the resulting photometry to the input values. In Figure 1, we show for the Chandra Deep Field-South (CDF-S, one of the deep Spitzer fields) the relative fraction of sources within $r \leq 2$ ". 3 (half the FWHM) and with a flux difference less than $50 \%$ compared to their input values. From these simulations, we estimated the flux-density limit where $80 \%$ of the input sources are recovered with this photometric accuracy; these are listed in Table 1. Simultaneously, we estimate that down to the $80 \%$ completeness limit the number of sources that result from fainter sources either by photometric errors or the merging of real sources is $\$ 10 \%$.

We also repeated the source detection and photometry process on the negative of each MIPS $24 \mu \mathrm{m}$ image. This test provides an estimate for the number of spurious sources arising from the noise properties of the image, as shown in Figure 1. For all of our fields, the spurious-source fraction for flux densities greater than the $80 \%$ completeness limit is less than $10 \%$.

\section{THE $24 \mu \mathrm{m}$ SOURCE COUNTS}

Figure 2 shows the $24 \mu \mathrm{m}$ cumulative and differential number counts that have been averaged over the fields listed in Table 1. The differential and cumulative counts (corrected and uncorrected) are listed in Table 2. The faintest datum (denoted by the open symbol in Fig. 2) is derived to the $50 \%$ completeness limit for the European Large-Area ISO Survey (ELAIS) field $\left(C_{50 \%}=35 \mu \mathrm{Jy}\right)$. The remaining (less deep)

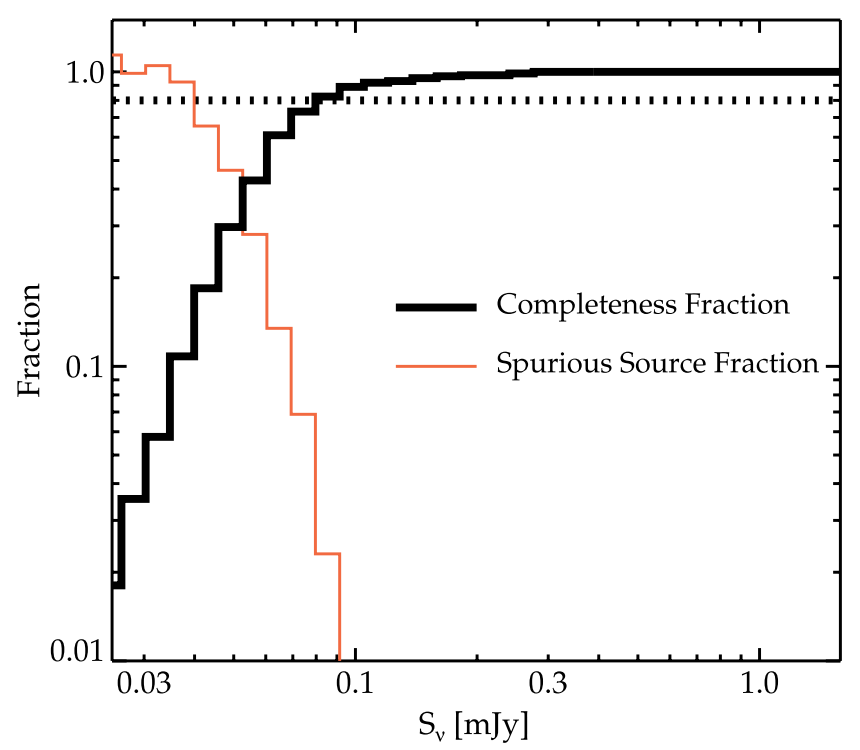

FIG. 1.- Reliability of source detection and photometry for the CDF-S, one of the deep Spitzer fields. The thick line shows the fraction of artificial sources detected in the image as a function of input flux density. For the CDF-S this fraction is $80 \%$ complete at $C_{80} \%=83 \mu \mathrm{Jy}$ (dotted line). The red line shows the ratio of the number of sources detected in a "negative" of the $24 \mu \mathrm{m}$ image to the number of "positive" sources as a function of flux density, which provides an estimate of the number of spurious sources due to noise features. 

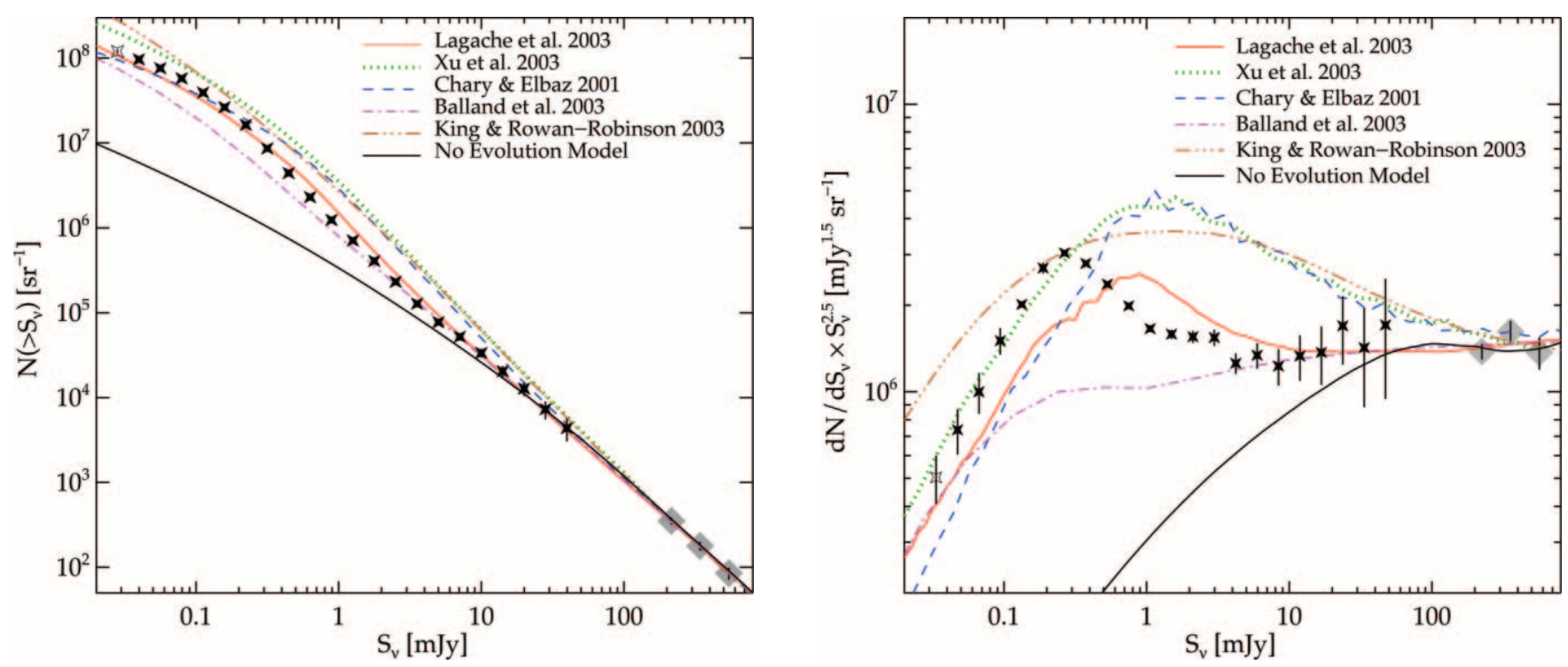

Fig. 2. - Cumulative (left) and differential (right) $24 \mu \mathrm{m}$ number counts. The differential counts have been normalized to a Euclidean slope, $d N / d S_{\nu} \sim S_{\nu}^{-2.5}$. The solid stars show the average counts from all the Spitzer fields (see Table 1) and corrected for completeness to their respective $80 \%$ limits. The open star corresponds to counts brighter than the $50 \%$ completeness limit from the ELAIS field. The error bars correspond to counting uncertainties and a cosmic-variance estimate based on the standard deviation of the field-to-field counts from the different fields. Each flux bin is $\Delta\left(\log S_{\nu}\right)=0.15$ dex. The shaded diamonds correspond to IRAS $25 \mu \mathrm{m}$ number counts from Hacking \& Soifer (1991) and adjusted assuming $\nu_{24} S_{\nu}(24 \mu \mathrm{m})=\nu_{25} S_{\nu}(25 \mu \mathrm{m})$. The curves show the predictions from various contemporary models from the literature (see figure inset; and adjusted slightly to match the observed IRAS counts) and a model based on the local ISO $15 \mu \mathrm{m}$ luminosity function and assuming nonevolving galaxy SEDs.

fields are used after correcting for completeness to the $80 \%$ level only. Error bars in the figure correspond to Poissonian uncertainties and an estimate for cosmic variance using the standard deviation of counts between the fields. For $S_{\nu} \leq$ $83 \mu \mathrm{Jy}$, where the counts are derived solely from the smaller ELAIS field, we estimate the uncertainty (18\%) using the standard deviation of counts at faint flux densities in cells of $130 \mathrm{arcmin}^{2}$ from the CDF-S (see below). We have ignored the contribution to the number counts from stars at $24 \mu \mathrm{m}$, which are negligible at these Galactic latitudes and flux densities based on preliminary Spitzer observations.

At bright flux densities, $S_{\nu} \gtrsim 5 \mathrm{mJy}$, the differential $24 \mu \mathrm{m}$ source counts increase at approximately the Euclidean rate, $d N / d S_{\nu} \sim S^{-2.5}$, which extends the trends observed by the IRAS $25 \mu \mathrm{m}$ population by 2 orders of magnitude (Hacking \& Soifer 1991; Shupe et al. 1998). For $S_{\nu} \simeq 0.4-4 \mathrm{mJy}$, the $24 \mu \mathrm{m}$ counts increase at super-Euclidean rates and peak near $0.2-0.4 \mathrm{mJy}$. This observation is similar to the trend observed in the ISO $15 \mu \mathrm{m}$ source counts (Elbaz et al. 1999), but the peak in the $24 \mu \mathrm{m}$ differential source counts occurs at fluxes fainter by a factor of $\approx 2.0$. The peak lies above the $80 \%$ completeness limit for nearly all fields and is seen in the counts of the fields individually. Thus, the observed turnover is quite robust. The counts converge rapidly with a subEuclidean rate at $\lesssim 0.2 \mathrm{mJy}$. We broadly fit the faint end $\left(S_{\nu} \simeq 35-130 \mu \mathrm{Jy}\right)$ of the differential number counts using a power law, $d N / d S_{\nu}=C\left(S_{\nu} / 1 \mathrm{mJy}\right)^{-\alpha}$, with $\alpha=-1.5 \pm 0.1$. This result is consistent with a separate analysis based solely on the ELAIS field (Chary et al. 2004).

The observed counts are strongly inconsistent with expectations from nonevolving models of the local IR-luminous population. In Figure 2, we show the $24 \mu \mathrm{m}$ counts derived from the local luminosity function at ISO $15 \mu \mathrm{m}$ (Xu 2000) and assuming local galaxy SEDs (Dale et al. 2001). We have used the local ISO $15 \mu \mathrm{m}$ luminosity function, because the $k$-correction between rest-frame ISO $15 \mu \mathrm{m}$ and observed MIPS $24 \mu \mathrm{m}$ bands is minimized at higher redshifts $(z \sim 0.6)$, which is more appropriate for the counts at fainter flux densities. However, using the local IRAS $25 \mu \mathrm{m}$ luminosity function (Shupe et al. 1998) yields essentially identical results.

TABLE 2

Measured Spitzer $24 \mu \mathrm{m}$ Number Counts

\begin{tabular}{|c|c|c|c|c|c|}
\hline $\begin{array}{c}\log S_{\nu} \\
(\mathrm{mJy}) \\
(1)\end{array}$ & $\begin{array}{c}d N / d S_{\nu} \\
\left(\mathrm{mJy}^{-1} \mathrm{sr}^{-1}\right) \\
(2)\end{array}$ & $\begin{array}{c}\delta\left(d N / d S_{\nu}\right) \\
\left(\mathrm{mJy}^{-1} \mathrm{sr}^{-1}\right) \\
(3)\end{array}$ & $\begin{array}{c}\log S_{\nu} \\
(\mathrm{mJy}) \\
(4)\end{array}$ & $\begin{array}{c}N\left(>S_{\nu}\right) \\
\left(\mathrm{sr}^{-1}\right) \\
(5)\end{array}$ & $\begin{array}{c}\delta\left(N\left(>S_{\nu}\right)\right) \\
\left(\mathrm{sr}^{-1}\right) \\
(6)\end{array}$ \\
\hline$-1.475 \ldots \ldots \ldots \ldots$ & $2.5(1.4) \times 10^{9}$ & $4.7(1.5) \times 10^{8}$ & -1.550 & $2.1(1.1) \times 10^{8}$ & $1.2(0.60) \times 10^{7}$ \\
\hline -1.325 ….............. & $1.5(1.2) \times 10^{9}$ & $2.7(0.97) \times 10^{8}$ & -1.400 & $1.2(0.92) \times 10^{8}$ & $7.6(4.9) \times 10^{6}$ \\
\hline$-1.175 \ldots \ldots \ldots \ldots \ldots \ldots$ & $8.7(6.9) \times 10^{8}$ & $1.4(1.4) \times 10^{8}$ & -1.250 & $9.6(7.2) \times 10^{7}$ & $3.7(0.68) \times 10^{6}$ \\
\hline
\end{tabular}

Notes.-Col. (1): Flux density for differential number counts. Cols. (2) and (3): Corrected differential counts and uncertainty. Col. (4): Flux density for cumulative number counts. Cols. (5) and (6): Corrected cumulative counts and uncertainty. Numbers in parentheses give the uncorrected values. Table 2 is published in its entirety in the electronic edition of the Astrophysical Journal Supplement. A portion is shown here for guidance regarding its form and content. 
While the nonevolving fiducial model is consistent with the observed $24 \mu \mathrm{m}$ counts for $S_{\nu} \gtrsim 20 \mathrm{mJy}$, it underpredicts the counts at $S_{\nu} \lesssim 0.4 \mathrm{mJy}$ by more than a factor of 10 .

Because the deep Spitzer fields are measured in many sight lines with large solid angle, we can estimate the fluctuations in the number of sources as a function of sky area and flux density in smaller sized fields. For example, in the CDF-S, which achieves an $80 \%$ completeness limit of $83 \mu \mathrm{Jy}$ over $\simeq 0.6 \operatorname{deg}^{2}$ (see Table 1 and Fig. 1), we have computed the variance in the number of sources in solid angles of 100 and $300 \operatorname{arcmin}^{2}$. For the sources that span the peak in the differential counts $(0.1-1 \mathrm{mJy})$, the fluctuation in the number of sources in flux-density bins of 0.15 dex is roughly $15 \%$ in these areas. This implies that small-sized fields suffer sizable field-to-field variation in the number of counts from the cosmic variance of source clustering. This effect is present in even larger fields: the number density of sources brighter than 0.3 mJy varies by $\sim 10 \%$ in fields of $\sim 0.5 \mathrm{deg}^{2}$ (Table 1 ) and is consistent with fluctuations expected from galaxy clustering on fields of this size at $z \sim 1$ (scale lengths of 20-70 Mpc). The counts presented here average over fields from many sight lines and significantly larger areas. We conservatively estimate that variations due to galaxy clustering correspond to uncertainties in the number counts of a less than a few percent in each flux bin.

\section{INTERPRETATION AND DISCUSSION}

The form of the observed $24 \mu \mathrm{m}$ source counts differs strongly from predictions of various contemporary models (see Fig. 2). Four of the models are phenomenological in approach, which parameterize the evolution of IR-luminous galaxies in terms of density and luminosity to match observed counts from ISO, radio, submillimeter, and other data sets. Several of these models (Chary \& Elbaz 2001; King \& Rowan-Robinson 2003; Xu et al. 2003) show a rapid increase in the number of sources at super-Euclidean rates at relatively bright flux densities $\left(S_{\nu} \gtrsim 10 \mathrm{mJy}\right.$ ) and peak near $1 \mathrm{mJy}$. These models generally predict a redshift distribution for the MIPS $24 \mu \mathrm{m}$ population that peaks near $z \sim 1$, based largely on expectations from the $I S O$ populations, and they overpredict the $24 \mu \mathrm{m}$ number counts by factors of $2-3$ at $\sim 1 \mathrm{mJy}$. The Lagache et al. (2003) model predicts a roughly Euclidean increase in the counts for $S_{\nu}>10 \mathrm{mJy}$. The shape of the counts in this model is similar to that of the observed distribution, but it peaks at $S_{\nu} \sim 1 \mathrm{mJy}$, at higher flux densities than the observed counts. This model predicts a redshift distribution that peaks near $z \sim 1$ but tapers slowly with a significant population of IR-luminous galaxies out to $z \gtrsim 2$ (Dole et al. 2003).

The model of Balland et al. (2003) is based on semianalytical hierarchical models within the Press-Schecter formalism, in which galaxies identified as "interacting" are assigned IR-luminous galaxy SEDs. This model includes additional physics in that the evolution of galaxies depends on their local environment and merger/interaction histories. Although this model predicts a near-Euclidean increase in the counts for $S_{\nu} \gtrsim$ $10 \mathrm{mJy}$, the counts shift to sub-Euclidean rates at relatively bright flux densities. The semianalytical formalism seems to not include important physics that are necessary to reproduce the excess of faint IR sources. This illustrates the need for large-area multiwavelength studies of Spitzer sources to connect optical- and IR-selected sources at high redshift to understand the mechanisms that produce IR-luminous stages of galaxy evolution.

The peak in the $24 \mu \mathrm{m}$ differential number counts occurs at fainter flux densities than that predicted from the phenome- nological models based on the ISO results. This may suggest possibilities such as a steepening in the slope of the IR luminosity function with redshift or evolution in the relation between the mid- and total IR. Phenomenological models that reproduce the IR background predict a faint-end slope of the IR luminosity function that should be quite shallow at high redshifts, with " $L^{*}$ " luminosities that correspond to $L_{\mathrm{IR}}>$ $10^{11} L_{\odot}$ for $z \gtrsim 1$ (see Hauser \& Dwek 2001). For most plausible IR luminosity functions, galaxies with $L^{*}$ luminosities dominate the integrated luminosity density. Elbaz et al. (2002) observed that the redshift distribution of objects with these luminosities in deep ISO surveys spans $z \simeq 0.8-1.2$, and that these objects constitute a large fraction of the total cosmic IR background. Therefore, it seems logical that objects with these luminosities dominate $24 \mu \mathrm{m}$ number counts at $0.1-$ $0.4 \mathrm{mJy}$, and it follows that their redshift distribution must lie at $z \sim 1-3$ (i.e., where this flux density corresponds to $\sim 10^{11}$ $10^{12} L_{\odot}$, using empirical relations from Papovich \& Bell 2002). Indeed, a similar conclusion is inferred on the basis of a revised phenomenological model using the $24 \mu \mathrm{m}$ number counts presented here (Lagache et al. 2004) and allowing for small changes in the mid-IR SEDs of IR-luminous galaxies. Examples of MIPS $24 \mu \mathrm{m}$ sources at these redshifts and luminosities have been readily identified in optical ancillary data (Le Floc'h et al. 2004). We therefore attribute the peak in the $24 \mu \mathrm{m}$ differential number counts at fainter flux densities to a population of luminous IR galaxies at redshifts higher than explored by ISO.

Integrating the differential source-count distribution provides an estimate for their contribution to the cosmic IR background at $24 \mu \mathrm{m}$, i.e., $I_{\nu}=\int d N / d S_{\nu} S_{\nu} d S_{\nu}$. For sources brighter than $60 \mu \mathrm{Jy}$, we derive a lower limit on the total background of $\nu I_{\nu}(24 \mu \mathrm{m})=1.9 \pm 0.6 \mathrm{nW} \mathrm{m}{ }^{-2} \mathrm{sr}^{-1}$. Because of the steep nature of the source counts, most of this background emission results from galaxies with fainter apparent flux densities. We find that $\simeq 60 \%$ of the $24 \mu \mathrm{m}$ background originates in galaxies with $S_{\nu} \leq 0.4 \mathrm{mJy}$, and therefore the galaxies responsible for the peak in the differential source counts also dominate the total background emission. Our result is consistent with the COBE DIRBE upper limit $\nu I_{\nu}(25 \mu \mathrm{m})<7 \mathrm{nW} \mathrm{m}^{-2} \mathrm{sr}^{-1}$ inferred from fluctuations in the IR background (Kashlinsky \& Odenwald 2000; Hauser \& Dwek 2001).

As a further estimate on the total $24 \mu \mathrm{m}$ background intensity, we have extrapolated the number counts for $S_{\nu}<$ $60 \mu \mathrm{Jy}$ using the fit to the faint-end slope of the $24 \mu \mathrm{m}$ number counts in $\S 3$. Under this assumption, we find that sources with $S_{\nu}<60 \mu \mathrm{Jy}$ would contribute $0.8_{-0.4}^{+0.9} \mathrm{nW} \mathrm{m} \mathrm{sr}^{-1}$ to the $24 \mu \mathrm{m}$ background, which when summed with the above measurement yields an estimate of the total background of $\nu I_{\nu}^{\text {(tot) }}(24 \mu \mathrm{m})=2.7_{-0.7}^{+1.1} \mathrm{nW} \mathrm{m}^{-2} \mathrm{sr}^{-1}$. For this value, the sources detected in the deep Spitzer $24 \mu \mathrm{m}$ surveys produce $\sim 70 \%$ of the total $24 \mu \mathrm{m}$ background.

We acknowledge our colleagues for stimulating conversations, the Spitzer Science Center staff for efficient data processing, Thomas Soifer and the IRS team for executing the Boötes-field observations, Daniel Eisenstein for cosmology discussions, Jim Cadien for his assistance with the data reduction, and the entire Spitzer team for their concerted effort. We also thank the referee, Matthew Malkan, for a thorough and insightful report. Support for this work was provided by NASA through contract 960785 issued by JPL/Caltech. 
Balland, C., Devriendt, J. E. G., \& Silk, J. 2003, MNRAS, 343, 107

Chary, R. R., \& Elbaz, D. 2001, ApJ, 556, 562

Chary, R. R., et al. 2004, ApJS, 154, 80

Dale, D. A., Helou, G., Contursi, A., Silbermann, N. A., \& Kolhatkar, S. 2001, ApJ, 549, 215

Dole, H., Lagache, G., \& Puget, J.-P. 2003, ApJ, 585, 617

Dole, H., et al. 2001, A\&A, 372, 364

Elbaz, D., Cesarsky, C. J., Chanial, P., Aussel, H., Franceschini, A., Fadda, D., \& Chary, R. R. 2002, A\&A, 384, 848

Elbaz, D., et al. 1999, A\&A, 351, L37

Fixsen, D. J., Dwek, E., Mather, J. C., Bennett, C. L., \& Shafer, R. A. 1998, ApJ, 508, 123

Franceschini, A., Aussel, H., Cesarsky, C. J., Elbaz, D., \& Fadda, D. 2001, A\&A, 378, 1

Gordon, K., et al. 2004, PASP, submitted

Hacking, P., \& Soifer, B. T. 1991, ApJ, 367, L49

Hauser, M. G. \& Dwek, E. 2001, ARA\&A, 39, 249

Hauser, M. G., et al. 1998, ApJ, 508, 25

Kashlinsky, A. \& Odenwald, S. 2000, ApJ, 528, 74

\section{REFERENCES}

King, A. J., \& Rowan-Robinson, M. 2003, MNRAS, 339, 260

Lagache, G., Dole, H., \& Puget, J.-L. 2003, MNRAS, 338, 555

Lagache, G., et al. 2004, ApJS, 154, 112

Le Floc'h, E., et al. 2004, ApJS, 154, 170

Madau, P. \& Pozzetti, L. 2000, MNRAS, 312, L9

Papovich, C. \& Bell, E. F. 2002, ApJ, 579, L1

Rieke, G. \& Low, F. 1972, ApJ, 176, L95

Rieke, G., et al. 2004, ApJS, 154, 25

Roussel, H., Sauvage, M., Vigroux, L. \& Bosma, A. 2001, A\&A, 372, 427

Shupe, D. L., Fang, F., Hacking, P. B., \& Huchra, J. P. 1998, ApJ, 501, 597

Soifer, B. T., \& Neugebauer, G. 1991, AJ, 101, 354

Soifer, B. T., Neugebauer, G., \& Houck, J. R. 1987, ARA\&A, 25, 187

Spinoglio, L., Malkan, M. A., Rush, B., Carrasco, L., \& Recillas-Cruz, E. 1995, ApJ, 453, 616

Stetson, P. B. 1987, PASP, 99, 191

$\mathrm{Xu}$, C. 2000, ApJ, 541, 134

Xu, C., Lonsdale, C. J., Shupe, D. L., Franceschini, A., Martin, C., \& Schiminovich, D. 2003, ApJ, 587, 90 